\title{
WEYL GROUP ACTIONS AND EQUIVARIANT HOMOTOPY EQUIVALENCE
}

\author{
KATSUO KAWAKUBO ${ }^{1}$
}

\begin{abstract}
Let $G$ be a compact Lie group and $G_{0}$ its identity component. Then we shall show that the normal representations of the corresponding fixed point components of $G$-homotopy equivalent manifolds are necessarily isomorphic when $G / G_{0}$ is a Weyl group of a compact connected Lie group.
\end{abstract}

1. Introduction. In [7] and [8], we defined an equivariant $J$-homomorphism $J_{G}$ : $K O_{G}(X) \rightarrow J_{G}(X)$ for a compact $G$-space $X$ and showed an equivariant analogue of Atiyah [2]. A corollary peculiar to the equivariant case is the following. The normal representations of the corresponding fixed point components of $G$-homotopy equivalent manifolds are stably homotopy equivalent.

In the present paper, we study the equivariant $J$-homomorphism in the case where $X$ is a point *. Let $G$ be a compact Lie group and $G_{0}$ its identity component. Then we shall show that $J_{G}: R O(G)\left(\cong K O_{G}(*)\right) \rightarrow J_{G}(*)$ is an isomorphism if $G / G_{0}$ is (for example) a Weyl group of a compact connected Lie group (Theorem 4.2).

By combining the above results, we have strong necessary conditions for two $G$-manifolds to be $G$-homotopy equivalent.

Namely the normal representations of the corresponding fixed point components of $G$-homotopy equivalent manifolds have to be isomorphic if $G / G_{0}$ is a Weyl group (Theorem 5.4).

Note that all the symmetric groups $S_{n}$ are realized as the Weyl groups of the unitary groups $U(n)$. Consequently $J_{S_{n}}: R O\left(S_{n}\right) \rightarrow J_{S_{n}}(*)$ is an isomorphism.

However $J_{G}: R O(G) \rightarrow J_{G}(*)$ is not an isomorphism in general for $G$ an alternating group $A_{n}$. We shall deal with $J_{A_{n}}$ in a forthcoming paper.

In [13], R. Schultz studied the relation between linear equivalence and topological equivalence of group representations. In $\$ 6$, we study the relations among linear equivalence, topological equivalence and stable homotopy equivalence of group representations.

The author wishes to thank Professor N. Kawanaka for enlightening him on the rationality of group representations. Thanks are also due to Professors A. Liulevicius and R. Schultz for kind advice.

Received by the editors May 22, 1979.

AMS (MOS) subject classifications (1970). Primary 55D10, 55E50; Secondary 20C15, 22 E55.

Key words and phrases. Compact Lie groups, equivariant $J$-homomorphism, Weyl groups, equivariant homotopy equivalence.

${ }^{1}$ Research supported in part by the Füjukai Foundation. 
2. Equivariant $J$-homomorphism. Let $G$ be a compact topological group and $X$ be a compact $G$-space. Let $\xi$ and $\eta$ be $G$-vector bundles over $X$. Denote by $S(\xi)$ (resp. $S(\eta))$ the sphere bundle associated with $\xi$ (resp. $\eta$ ).

DefinItion 2.1. $S(\xi)$ and $S(\eta)$ are said to be of the same $G$-fiber homotopy type if there exist fiber-preserving $G$-maps

$$
f: S(\xi) \rightarrow S(\eta), \quad f^{\prime}: S(\eta) \rightarrow S(\xi),
$$

and fiber-preserving $G$-homotopies,

$$
h: S(\xi) \times I \rightarrow S(\xi), \quad h^{\prime}: S(\eta) \times I \rightarrow S(\eta),
$$

with

$$
\begin{array}{ll}
h \mid S(\xi) \times 0=f^{\prime} \cdot f, & h \mid S(\xi) \times 1=\text { identity } \\
h^{\prime} \mid S(\eta) \times 0=f \cdot f^{\prime}, & h^{\prime} \mid S(\eta) \times 1=\text { identity. }
\end{array}
$$

Let $K O_{G}(X)$ be the Grothendieck-Atiyah-Segal group [3] defined in terms of real $G$-vector bundles over $X$. Let $T_{G}(X)$ be the additive subgroup of $K O_{G}(X)$ generated by elements of the form $[\xi]-[\eta]$, where $\xi$ and $\eta$ are $G$-vector bundles whose associated sphere bundles are $G$-fiber homotopy equivalent.

Definition 2.2. We define our equivariant $J$-group $J_{G}(X)$ by $J_{G}(X)=$ $K O_{G}(X) / T_{G}(X)$ and define our equivariant $J$-homomorphism $J_{G}$ by the natural epimorphism $J_{G}: K O_{G}(X) \rightarrow J_{G}(X)$.

Our equivariant $J$-homomorphism is an equivariant version of the $J$-homomorphism of Adams [1]. Precisely speaking, $J_{G}(X) \cong J(X)$ and $J_{G}: K O_{G}(X) \rightarrow J_{G}(X)$ coincides with $J: K O(X) \rightarrow J(X)$ of Adams [1] when $G$ is the trivial group.

3. Equivariant Adams operations. In this section we recall some results in [4] and [12]. Denote by $Q$ the rational number field. Let $G$ be a finite group of order $N$ and let $\Gamma_{N}$ be the Galois group of $Q(\omega)$ over $Q$ where $\omega$ is a primitive $N$ th root of unity. It is well known that $\Gamma_{N}$ is isomorphic to the multiplicative group $Z_{N}^{*}$ of units in the ring $Z_{N}$. Denote by $R O(G)$ the real (orthogonal) representation ring of $G$. Then $\Gamma_{N}$ acts on $R O(G)$ in two manners. For $s \in Z_{N}^{*}\left(\cong \Gamma_{N}\right)$ and for a $G$-representation $V$, the equivariant Adams operation is defined by

$$
\Psi^{s}(V)=P_{s}\left(\Lambda^{1} V, \Lambda^{2} V, \ldots, \Lambda^{n} V\right)
$$

where $P_{s}\left(\sigma_{1}, \ldots, \sigma_{n}\right)=x_{1}^{s}+\cdots+x_{n}^{s}, \sigma_{i}$ the $i$ th elementary symmetric function of $x_{1}, \ldots, x_{n}, n>\operatorname{dim} V$, and $\Lambda^{i} V$ is the $i$ th exterior power of $V$.

On the other hand, it is well known (see for example [6]) that $V$ is realized by a homomorphism $\rho: G \rightarrow \mathrm{GL}(\operatorname{dim} V, Q(\omega))$. For $s \in Z_{N}^{*}$, let $\alpha^{s}$ be the element of the Galois group $\Gamma_{N}$ defined by $\alpha^{s}(\omega)=\omega^{s}$. Then $\Psi^{s} V$ is associated to the homomorphism $\bar{\alpha}^{s} \circ \rho$ where $\bar{\alpha}^{s}: \mathrm{GL}(\operatorname{dim} V, Q(\omega)) \rightarrow \mathrm{GL}(\operatorname{dim} V, Q(\omega))$ is induced by $\alpha^{s}$. It is shown in [4] and [12] that the above two definitions of $\Psi^{s}$ coincide.

Definition 3.1. Let $W O(G)$ be the subgroup of $R O(G)$ generated by the $V-\Psi^{s} V$ where $V \in R O(G), s \in Z_{N}^{*}$.

Definition 3.2. Let $W O(G)^{\prime}=\left\{V-W \in R O(G) \mid \operatorname{dim} V^{H}=\operatorname{dim} W^{H}\right.$ for all subgroups $H$ of $G\}$ where $V^{H}=\{v \in V \mid h v=v$ for all $h \in H\}$ and similar for $W^{H}$. 
Then Lee and Wasserman showed the following proposition on which our results will be based.

Proposition 3.3 (Proposition 3.17 of [12]). For any finite group, $W O(G)=$ $W O(G)^{\prime}$.

4. Groups $G$ for which $J_{G}$ is an isomorphism. In this section, we consider $J_{G}$ : $K O_{G}(X) \rightarrow J_{G}(X)$ in the case where $X$ is a point *. Let $G$ be a compact Lie group and $G_{0}$ its identity component.

LEMMA 4.1. If all real representations of $G / G_{0}$ have rational characters, then $J_{G}$ : $R O(G)\left(\cong K O_{G}(*)\right) \rightarrow J_{G}(*)$ is an isomorphism.

Proof. We show that $T_{G}(*)=\{0\}$. Let $V-W$ be an arbitrary element of $T_{G}(*)$. We may suppose that the unit spheres $S(V)$ and $S(W)$ are $G$-homotopy equivalent. Denote by $V^{G_{0}}$ (resp. $W^{G_{0}}$ ) the fixed point set of $G_{0}$ and by $V_{G_{0}}\left(\right.$ resp. $W_{G_{0}}$ ) its orthogonal complement in $V$ (resp. $W$ ). According to [16], $S(V)$ and $S(W)$ are $G$-homotopy equivalent if and only if the following two conditions hold.

(1) $S\left(V^{G_{0}}\right)$ and $S\left(W^{G_{0}}\right)$ are $G / G_{0}$-homotopy equivalent.

(2) $V_{G_{0}}$ and $W_{G_{0}}$ are isomorphic $G$-representations.

Hence $V^{G_{0}}-W^{G_{0}}$ belongs to $T_{G / G_{0}}(*)$. In particular, $\operatorname{dim} S\left(V^{G_{0}}\right)^{H}=$ $\operatorname{dim} S\left(W^{G_{0}}\right)^{H}$ for all subgroups $H$ of $G / G_{0}$. Namely $V^{G_{0}}-W^{G_{0}}$ belongs to $W O\left(G / G_{0}\right)^{\prime}$. It follows from Proposition 3.3 that $V^{G_{0}}-W^{G_{0}}$ also belongs to $W O\left(G / G_{0}\right)$. It is Galois theory that the elements of $R O\left(G / G_{0}\right)$ invariant under $\Gamma_{N}$ consist precisely of those virtual representations with rational characters where $N$ is the order of the group $G / G_{0}$. Therefore $W O\left(G / G_{0}\right)=\{0\}$ by the assumption of Lemma 4.1. It follows that $V^{G_{0}}-W^{G_{0}}=0$ in $R O\left(G / G_{0}\right)$. Accordingly $V^{G_{0}}-$ $W^{G_{0}}=0$ in $R O(G)$.

On the other hand $V_{G_{0}}-W_{G_{0}}=0$ in $R O(G)$ (see (2) above). Thus we have that $V-W=0$ in $R O(G)$.

This completes the proof of Lemma 4.1.

THEOREM 4.2. If $G / G_{0}$ is a Weyl group of a compact connected Lie group, then $J_{G}$ : $R O(G) \rightarrow J_{G}(*)$ is an isomorphism.

Proof. The characters of complex (unitary) representations of a Weyl group of a simple Lie group are rational valued. This fact was shown by A. Young [17] for the families of groups of type $A_{l}$ and $B_{l}$, by W. Specht [15] for the family of groups of type $D_{l}$, by T. Kondo [11] for the group of type $F_{4}$ and by M. Benard [5] for the groups of type $E_{6}, E_{7}$ and $E_{8}$. This result is easily shown for the group of type $G_{2}$, which is dihedral of order 12. In fact, they showed stronger results as follows. Any irreducible character of the above groups has Schur index 1 over $Q$, i.e., any complex representation of the above groups is a rational representation.

It is easy to see that a Weyl group of a compact connected Lie group is isomorphic to the direct product of Weyl groups of several simple Lie groups. Note that an irreducible complex representation of the direct product $G_{1} \times G_{2}$ of finite groups $G_{1}, G_{2}$ is the tensor product of some irreducible representations of $G_{1}$ and $G_{2}$ (see for example [14]). Hence a complex representation of $G_{1} \times G_{2}$ is rational 
valued if all irreducible representations of $G_{1}$ and $G_{2}$ are rational valued.

Thus we have that the characters of complex representations of a Weyl group of a compact connected Lie group are rational valued. If $\chi$ is the character of a real representation $V$, then the complexification $c V=C \otimes_{R} V$ has the same character $\chi$. This implies the character of a real representation of $G / G_{0}$ is also rational valued. Hence Theorem 4.2 follows from Lemma 4.1.

5. A necessary condition for $G$-homotopy equivalence. We first recall the following

THEOREM 5.1 ([7], [8]). Let $G$ be a compact Lie group and $M_{1}, M_{2}$ be closed smooth $G$-manifolds with tangent bundles $T M_{1}, T M_{2}$ respectively. Let $f: M_{1} \rightarrow M_{2}$ be a $G$-homotopy equivalence. Then we have $J_{G}\left(\left[T M_{1}\right]\right)=J_{G}\left(\left[f^{*} T M_{2}\right]\right)$.

Let $f: M_{1} \rightarrow M_{2}$ be a $G$-homotopy equivalence. Denote by $F_{1}^{\mu}$ each component of the fixed point set of $M_{1}$. Set $F_{2}^{\mu}=f\left(F_{1}^{\mu}\right)$. Then $F_{2}^{\mu}$ is a component of the fixed point set of $M_{2}$ and the union $\cup_{\mu} F_{2}^{\mu}$ is exactly the fixed point set of $M_{2}$. Denote by $N_{i}^{\mu}$ the normal bundles of $F_{i}^{\mu}$ in $M_{i}(i=1,2)$. Then we have

Corollary 5.2. $J_{G}\left(\left[N_{1}^{\mu}\right]\right)=J_{G}\left(\left[\left(f \mid F_{1}^{\mu}\right)^{*} N_{2}^{\mu}\right]\right)$.

Let $V_{i}^{\mu}$ be the normal representations of $F_{i}^{\mu}$ in $M_{i}(i=1,2)$. Regarding $V_{i}^{\mu}$ as $G$-vector bundles over one point, we have in particular

Corollary 5.3. $J_{G}\left(\left[V_{1}^{\mu}\right]\right)=J_{G}\left(\left[V_{2}^{\mu}\right]\right)$.

By combining Theorem 4.2 and Corollary 5.3, we obtain a necessary condition for two $G$-manifolds to be $G$-homotopy equivalent.

THEOREM 5.4. If $G / G_{0}$ is a Weyl group of a compact connected Lie group, then $V_{1}^{\mu}$ and $V_{2}^{\mu}$ are isomorphic $G$-representations.

6. Relations among linear equivalence, topological equivalence and stable homotopy equivalence. In [13], R. Schultz gave some classes of groups for which linear equivalence equals topological equivalence. Among the classes of Corollary 2.3 in [13], even stable homotopy equivalence equals linear equivalence [9] for (i) and (iv) where

(i) all groups of the form $\left(Z_{2}\right)^{k}, k \geqslant 1$,

(iv) all groups of the form $Z_{4} \times\left(Z_{2}\right)^{k}$.

On the contrary, homotopy equivalence does not equal linear equivalence for all cyclic groups $Z_{n}$ with $n \neq 1,2,3,4,6$ [9].

More generally, stable homotopy equivalence equals linear equivalence for a compact Lie group $G$ if $G / G_{0} \cong$ a Weyl group or if each cyclic subgroup of $G / G_{0}$ is of order 1, 2, 3, 4 or 6 [10]. Examples are the following classes of compact Lie groups $G$.

(i) $G / G_{0} \cong\left(S_{n}\right)^{k},\left(A_{4}\right)^{k},\left(D_{3}\right)^{k},\left(D_{4}\right)^{k}$ where $n, k \geqslant 1$ and $D_{n}$ are dihedral groups.

(ii) $G / G_{0} \cong\left(Z_{2}\right)^{j} \times\left(Z_{3}\right)^{k},\left(Z_{2}\right)^{j} \times\left(Z_{4}\right)^{k}$, where $j, k \geqslant 0$.

(iii) $G \cong \Pi_{i} O\left(a_{i}\right) \times \Pi_{j} \operatorname{Pin}\left(b_{j}\right) \times \Pi_{k} \operatorname{Pin}^{c}\left(c_{k}\right) \times \Pi_{m} N U\left(d_{m}\right)$,

where $N U(n)$ denotes the group consisting of all unitary or conjugate-linear unitary maps of $\mathbf{C}^{n}$ and $a_{i}, b_{j}, c_{k}$ and $d_{m}$ are arbitrary nonnegative integers. 


\section{REFERENCES}

1. J. F. Adams, On the groups $J(X)-I$, Topology 2 (1963), 181-195.

2. M. F. Atiyah, Thom complexes, Proc. London Math. Soc. 11 (1961), 291-310.

3. M. F. Atiyah and G. B. Segal, Equivariant $K$-theory, University of Warwick, 1965 (mimeographed note).

4. M. F. Atiyah and D. O. Tall, Group representations, $\lambda$-rings and the J-homomorphism, Topology 8 (1969), 253-297.

5. M. Benard, On the Schur indices of characters of the exceptional Weyl groups, Ann. of Math. (2) 94 (1971), 89-107.

6. C. W. Curtis and I. Reiner, Representation theory of finite groups and associative algebras, Interscience, New York, 1962.

7. K. Kawakubo, G-homotopy equivalent manifolds and $J_{G}$-homomorphism, Proc. Japan Acad. 54 (1978), 104-106.

8. , Compact Lie group actions and fiber homotopy type, J. Math. Soc. Japan (to appear).

9. __ Equivariant homotopy equivalences of group representations, J. Math. Soc. Japan 32 (1980), $105-118$.

10. Cancellation law for G-homotopy equivalent representations (to appear).

11. T. Kondo, The characters of the Weyl group of type $F_{4}$, J. Fac. Sci. Univ. Tokyo Sect. IA Math. 11 (1965), 145-153.

12. C. N. Lee and A. G. Wasserman, On the groups JO(G), Mem. Amer. Math. Soc. No. 159 (1975).

13. R. Schultz, On the topological classification of linear representations, Topology 16 (1977), 263-269.

14. J. P. Serre, Représentations linéaires des groupes finis, Hermann, Paris, 1971.

15. W. Specht, Eine Verallgemeinerung der symmetrischen Gruppe, Schr. Math. Sem. Berlin 1 (1932), $1-32$.

16. P. Traczyk, On the G-homotopy equivalence of spheres of representations, Math. Z. 161 (1978), 257-261.

17. A. Young, Quantitative substitutional analysis. IV, V, Proc. London Math. Soc. (2) 31 (1930), 253-272; 273-288.

Department of Mathematics, Osaka University, Toyonaka, Osaka, JaPan 